\title{
The generation game
}

\section{Proteomics is hungry for well-validated antibodies. Nathan Blow looks at the options and sees how researchers are redefining the way to generate an antibody.}

The products of more than 22,700 genes make up the human proteome. But researchers hoping to unpick the mysteries of the proteome are restricted by the fact that antibodies against only a small percentage of these proteins are available. Although commercial production of antibodies is well established, the market has so far been driven by the popularity of particular antibody targets. More than 1,000 antibodies against the tumour suppressor p53 are available, for example, but the less sought-after targets frequently have none.

With the rise of proteomics, the need for antibodies has become global and is no longer limited to a small number of targets central to most hypothesis-driven research projects. "One of the hurdles in proteomics is a lack of high-quality, well characterized affinity reagents," says Henry Rodriguez, director of the clinical proteomic technologies initiative for cancer at the US National Cancer Institute (NCI) in Bethesda, Maryland.

Not only do researchers need access to more antibodies, they also need to know how these antibodies have been characterized to determine whether they will work in the assay they are using. "Large numbers of antibodies are already available, but an investigator has to navigate through a complex system to find out which target antibodies are going to be appropriate for his or her particular assays," says Adam Clark, who works on the NCI's proteomic technologies initiative.

\section{Standard issue}

At present, there is no universal validation: an antibody that works wonders for a Western blot may perform poorly in immunohistochemistry. This growing need for faster antibody production and stronger validation data is leading many groups to explore highthroughput methodologies for creating and validating affinity reagents.

A group led by Mathias Uhlén at the Royal Institute of Technology in Stockholm, Sweden, is spearheading an initiative to assemble the Human Protein Atlas (www.proteinatlas.org). The project aims to explore the entire human proteome using antibodies. Uhlén's group is producing polyclonal antibodies directed against each human protein, and then characterizing the antibodies using Western blots, protein microarrays and immunohistochemistry.

The project generates around ten new polyclonal antibodies and more than 10,000 immunohistochemistry images every day - an achievement that relies heavily on highthroughput methodology. Although most steps in the process are amenable to automation, some, such as annotating tissue immunohistochemistry images, are proving to be a significant challenge. Indeed, analysing these images still involves ten pathologists who have to annotate them manually. "All the annotation is Internet based. The pathologists view and evaluate 600 tissue images per antibody via a web-based tool on their personal computers, and the results are stored in our database," says Uhlén.

In addition to producing and testing their own antibodies,

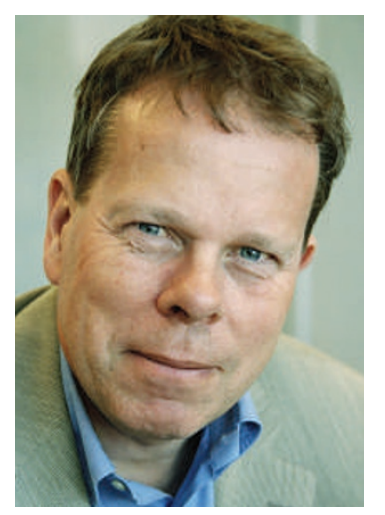

Mathias Uhlén is using antibodies to unravel the human proteome. ofeach antibody generated by the atlas will be of each antibody generated by the atlas will be made available through Atlas Antibodies of Stockholm.

Other initiatives are focusing on monoclonal, rather than polyclonal, antibodies against proteins on a large scale. In 2002, for example, the Wellcome Trust Sanger Institute near Cambridge, UK, launched the Atlas of Gene Expression. Although a change in focus at the institute means that this is closing down, the atlas was set up to generate high-quality monoclonal antibodies that were well characterized for a variety of assays. Much as for the Human Protein Atlas, a well-standardized characterization was key. "Historically, the validation of antibodies has been ad hoc with different people generating antibodies that have been assessed in different ways," says project leader John McCafferty.

Uhlén and his team will put antibodies from commercial sources through their standardized quality-control pipeline. Uhlén says he was surprised that only about $35 \%$ of commercial antibodies seemed to work - although he notes that this could be a result of the way his group analyses them. "We decided to use a very standardized way of validating antibodies: if they don't work, we don't try other ways of doing it," he says. The success rate may be low, but such a standardized quality-control process
The project involved four key groups. One generated the proteins of interest and did quality control. This team used the Gateway cloning system made by Invitrogen of Carlsbad, California, to move a variety of open reading frames between different expression vectors and so improve yields for troublesome proteins. A second group used phage display for high-throughput screening of a single-chain antibody library generated at the Sanger Institute and containing more than $10^{10}$ phage 
clones. The other two groups were dedicated to immunohistochemistry and the informatics infrastructure necessary to deal with the large volume of data.

To deal with image acquisition issues, the institute collaborated with Applied Imaging of San Jose, California, to develop an automated high-throughput image-analysis system suitable for tissue microarray applications. So far, the Sanger project has generated more than 4,000 monoclonal antibodies to 290 antigens, which are available to buy from Geneservice in Cambridge, UK.

Although the project is being discontinued, McCafferty says that much has been learned about the bottlenecks of high-throughput generation of antibodies and how these can be overcome. "Surprisingly, the generation of the antibodies was not the major issue," he says. "The bottlenecks were generating good quality protein product to do selection, and how to deal with the large amounts of image data a project such as this produces."

\section{Finding affinity}

Even as the Sanger project comes to a close, other initiatives are beginning to gather steam - although these have been hampered somewhat by a lack of funding. "There seems to be a reluctance from the funding agencies to put money into large-scale antibody initiatives," says Andrew Bradbury of the biosciences division at Los Alamos National Laboratory in New Mexico. The NCI's five-year, \$104-million clinical proteomic technologies initiative



Cells stained with the 4G10 anti-phosphotyrosine antibody from Millipore.

that is now getting off the ground may be the start of a change.

In 2005, the NCI held a workshop to discuss affinity capture. It found that the scientific community wanted renewable resources that were well characterized for performance data, says Clark. The meeting also revealed that the community was concerned by the lack of characterization data for most available antibodies.

Following this lead, the NCI proteomics reagent core, one of the centres in the clinical proteomic technology initiative, is embarking on the production of affinity reagents. To focus its efforts, it has identified a list of protein targets: all cancer-related proteins for which no com- mercial antibodies are yet available. The core will develop monoclonal antibodies that will be characterized by Western blots, enzyme-linked immunosorbent assay (ELISA), immunohistochemistry and immunoprecipitation followed by mass spectrometry. "All the raw data on how the antibodies perform on a variety of assays will be provided and an investigator will be able to acquire these antibodies through a website organized by the NCI," says Clark.

In Europe, another group of investigators plans to generate affinity reagents against the human proteome. The group, called ProteomeBinders, consists of 26 European Union and two US institutional partners. "The goal or the hope is to get funding from the European Union to put a project together next year or the year after," says Bradbury, one the US participants.

Although the antibody remains the affinity reagent of choice, the exploration of alternative binders by large groups, such as ProteomeBinders, shows how far these non-traditional reagents have come in a relatively short time.

\section{Gold standard}

A quick glance through the catalogues from commercial vendors and researchers reveals thousands of antibodies not only to proteins, but also to specific protein changes such as post-translational modifications. Still other companies offer to produce antibodies to an investigator's antigen of interest.

Monoclonal antibodies produced by animal immunization remain the 'gold standard' of

\section{ANTIBODIES IN THE FAST LANE}

In making recombinant antibodies, the resulting antibody is only as good as the combinatorial library and the screening assay. The trick is to find the molecule of highest affinity and specificity for the target among a library of millions of clones. Traditionally, recombinant antibody libraries have been phage-based and the screening relied on enzyme-linked immunosorbent assay (ELISA), not a high-throughput method. Changes in both phage display and screening methods are now moving recombinant antibody production into a high-throughput world.

Flow cytometry has become the assay of choice for rapid screening of clones from recombinant antibody libraries. “We looked at different ways of screening. Flow cytometry was the only one that seemed to meet our throughput requirements," says Andrew Bradbury of Los Alamos National Laboratory in New Mexico.
Bradbury's group has developed a flow-cytometry assay to screen its single-chain antibody-fragment phage libraries using a mixture of beads coated with specific and non-specific antigens. The method rapidly identifies antibodies that have good affinity for the protein of interest while discarding those that show low specificity.

Phage-display screening methods are also used to identify antibodies that target posttranslational modifications (PTMs) such as phosphorylation or acetylation. As PTMs have a role in many processes - from gene regulation to apoptosis they are of growing interest for the biological community. Companies have responded by developing antibodies targeting proteins in a specific state of modifications. "Antibodies to PTMs are gaining in importance with customers," says Kumar Bala, director of antibody technologies for Millipore in the company's lab in Temecula, California.

But obtaining antibodies directed against PTMs is not a trivial task. Rockland Immunochemicals of Gilbertsville, Pennsylvania, has put in a lot of effort to develop antibodies for looking at phosphorylated and nonphosphorylated forms of various proteins in a sequence independent context, says Daniel O'Shannessy,

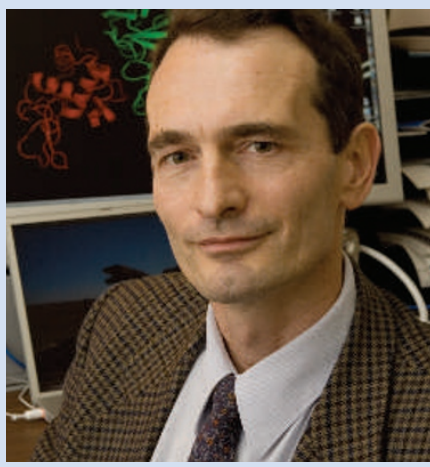

Andrew Bradbury develops recombinant single-chain antibodies. the company's vice-president of corporate development.

Antibodies that recognize PTMs independently of the protein site on which the modification occurs are useful - particularly for enriching, for example, all phosphorylated proteins from a cell. But such antibodies are hard to make by animal immunization as the PTM itself is not immunogenic. So scientists are turning to recombinant molecules and in vitro screening such as phage display to isolate 'pan-PTM' affinity reagents.

Although making steps in the right direction, many more antibodies and further improvements in affinity reagent technology will be needed to understand and characterize the full range of PTMs found in nature. Still, Bala argues that the "best tools for purifying, identifying, differentiating and characterizing PTMs are antibodies". 
affinity reagents. They are relatively renewable, can usually be made with high specificity and affinity for their target and can be used in common biochemical assays such as Western blotting, ELISA and immunochemistry. But the traditional monoclonal antibody has its drawbacks. Its production can be challenging, timeconsuming and costly. So there is a lot of interest in identifying novel affinity reagents that would be less expensive and quicker to produce. "The future is with alternative binders," says Bradbury, who works with single-chain antibodies (see 'Antibodies in the fast lane', page 743).

\section{Optimistic expression}

The structural characteristics of antibodies make it difficult to produce recombinant versions in bacteria and restricts their use in some high-throughput screening methods. But George Georgiou and his colleagues at the University of Texas at Austin have come up with a method to produce and screen fulllength immunoglobulin $\mathrm{G}$ (IgG) antibodies expressed in Escherichia coli (Y. Mazor et al. Nature Biotechnol. 25, 563-565; 2007).

The technology produces full-length antibodies that are initially tethered to the inner membrane of the bacterium. When the bacteria are treated with EDTA and lysozyme, the resulting spheroplasts with exposed antibodies can be selected in a high-throughput manner by using fluorescently labelled antigens and flow cytometry.

"We can isolate several bacterial clones expressing full-length IgG antibodies that can bind to the antigen with the requisite affinity," says Georgiou. "The advantage of the technology is that the expression of the antibodies is directly in bacteria and we can use the bacteria to produce the antibody without going through the steps of reformatting the antibody and then expressing in a mammalian system." But

the antibodies are not glycosylated, which limits some of the therapeutic applications.

The more traditional way to screen and obtain IgG antibodies rapidly is the generation of recombinant antibody fragments. Single-chain variable $(\mathrm{scFv})$ antibody fragments are created by the fusion of the variable regions of the heavy and light chains of immunoglobulins using a short peptide linker. This allows scFv fragments to be expressed from a single open reading frame and screened by phage display or other highthroughput approaches.

Although larger than $\mathrm{scFv}$ fragments and still composed of two independent polypeptide chains, Fab antibody fragments are also being used and are usually favoured for their high stability and compatibility with existing antibody-based assays. The Fab antibody

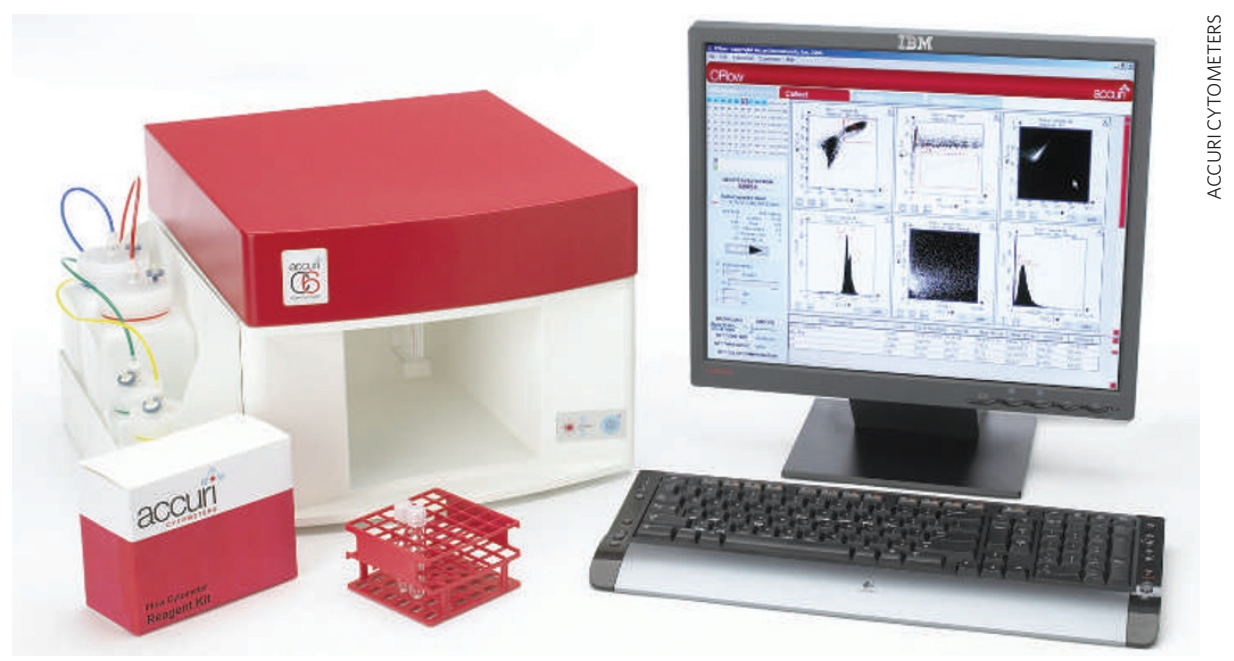

Flow cytometry is the assay of choice for screening recombinant-antibody libraries.

region is the antigen-binding region of the immunoglobulin. Fab fragments consist of one constant and one variable domain from each of the heavy and light chains.

Both scFv- and Fab-based technologies are developing rapidly, with several companies now supplying either $s c F v$ or Fab libraries and screening systems to consumers. One such company is BioInvent of Lund, Sweden, which provides the $\mathrm{n}-\mathrm{CoDeR}$ human-antibody library based on both the scFv and Fab formats. Other companies, including Cambridge Antibody Technology in Cambridge, UK, and MorphoSys in Martinsried, Germany, have developed human-derived phage-display libraries using either the scFv or Fab format to identify binding regions for development of therapeutic monoclonal antibodies.

Although rapidly generated and effective for many in vitro applications, scFv and Fab fragments are less effective for therapeutic applications because they have short half-lives. "Single-chain fragments can't really be used in animals because they are cleared very rapidly - the half-life of a single chain Sv is about 10 minutes, whereas full-length antibodies can persist for several days," says Georgiou.

\section{Breaking with tradition}

Described for the first time in 1997 by Uhlén and his colleagues, affibodies were among the first non-immunoglobulinbased affinity reagents. These small molecules are based on a bacterial receptor (Staphylococcus aureus protein $\mathrm{A}$ ), and use combinatorial protein engineering to introduce random mutations in the affinity region. Affibody of Bromma in Sweden, which was co-founded by Uhlén, currently produces affibody-based reagents for basic research laboratories and commercial partners.
Another non-immunoglobulin-based affinity reagent that is becoming more widely used is the aptamer. Made of DNA, RNA or modified nucleic acids and typically 15-40 bases in length, aptamers have a stable tertiary structure that permits protein binding through van der Waals forces, hydrogen bonding and electrostatic interactions. Early studies showed that aptamers can be highly specific for target proteins, with the ability to distinguish between related members of a protein family (S. D. Seiwart et al. Chem. Biol. 7, 833843; 2000).

Unlike the scFv and Fab fragments, both aptamers and affibodies are useful for in vivo applications because they have longer halflives. In addition, both function well in the reducing environment of the cell cytosol, which is a problem for larger monoclonal antibodies. Currently, Affibody is testing an HER2-binding affibody as an alternative to herceptin for treatment of HER-2-positive breast cancer with a clinical proof-of-principle microdosing study to occur this year.

Archemix in Cambridge, Massachusetts, has three aptamer-based therapeutics in phase I clinical trials. Two aptamers target coagulation processes and the third targets nucleolin, a protein that is involved in the development of some cancers.

Overall, there is much optimism regarding the future of alternative affinity reagents. But several problems have to be overcome before they are adopted more widely by the scientific community. One of the most pressing issues is the inability to produce these reagents at a truly high-throughput scale. Overcoming this obstacle would make these alternative binders not only cheaper to produce than traditional antibodies, but would also require significantly less time. "I would love to see a major technology breakthrough where someone shows that you can actually produce these in a high-throughput manner, but so far I don't think anyone has been able to do that," says Uhlén.

Nathan Blow is the technology editor for Nature and Nature Methods. 